Visualizing Objects, Places, and Spaces: A Digital Project Handbook

\title{
Budgets \& Funding for Dimensional Projects
}

Beth Fischer, Hannah Jacobs, Jayde Rice

Published on: Oct 14, 2019

DOI: 10.21428/51bee781.023eebc0

License: Creative Commons Attribution 4.0 International License (CC-BY 4.0). 
A professional-looking dimensional model can be made

entirely with tools you already have, using programs that are free for educational use. Complex dimensional projects can run into thousands of dollars, though, if you need to acquire equipment, hire experts, or pay for software.

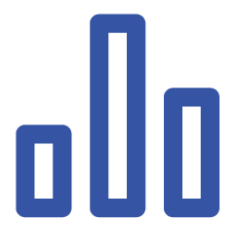

\section{Photogrammetry}

If you are working on a project using photogrammetry, the most common factor affecting the costs is the way you acquire the images you use to build your model. If you are modeling an object and you have full access to it, and you already have a camera with manual control, you may not need to spend any money out of pocket at all. On the other hand, if you need to model a building, you may need to buy a drone or pay for scaffolding, and you also may need to pay for permits to allow access.

Here are some ideas for how to budget for the costs of photogrammetry, and how you can limit the expense:

- Camera: You can typically use any consumer-grade dSLR camera, which you can often borrow from a friend if you don't have one yourself. You can even use a smartphone with a camera app that has manual control. These apps are often free for limited use, and will be under $\$ 5$ if you need to buy one. If you need to buy a full dSLR camera, you can find a reliable model for $\$ 400-\$ 500$. If you are using a drone, some drones have built in cameras.

- Permits or cost for access to objects/space: Make sure you check whether you have any costs for access to the thing you are modeling. This might mean permits from the location for buildings, or permits for setting up equipment; for objects, it might be a fee for reproduction or a research access fee or something similar.

- Tripod: While this is not an absolute necessity, a tripod will help you stabilize the camera so that you can get sharp photos. This can also help speed up the process and ensure that you get consistent photos from each height. These can often be borrowed or rented, or you can buy an entry-level one for $\$ 25-\$ 50$.

- Remote shutter release: This is another optional piece of equipment that can save you a lot of time and help ensure that you get sharp photos, especially if you are 
working by yourself. These are fairly cheap, usually around $\$ 30$ for a dSLR and under \$20 for a smartphone camera.

\section{- Additional equipment for photogrammetry of objects:}

- Turntable: While not absolutely necessary, this will save you a lot of headaches as you try to take photos of objects from all angles. With a turntable, you can set up your camera and quickly get all the photos from a consistent height around the object. You can find one for under $\$ 15$ online -- look for a white one or put a white cloth over it. These are sometimes called "lazy susans".

- Light tent: This will help you keep a plain background on your object, and help diffuse the light so that you don't get shadows. You can buy one for $\$ 20$, or you can make one yourself using white sheets.

- Lights: You want to have even light with as few shadows as possible, so you will often need extra lights. Simple clip-on task lights sold in big box or hardware stores are great for this, though you can use lights specially designed for photography if you have access to them.

- For photogrammetry of buildings, you also need a way to get photos from above. Consider:

- A drone: this has become the most common way people get photos from above buildings for photogrammetry. While you need to make sure you have the necessary permissions and be very careful to follow laws about their use, they have become fairly affordable: a high-quality option for this purpose will be under $\$ 1,000$. You need a drone where you can control the intervals for taking photos or control the shutter from the ground, but as you won't be taking photos from a great height above the building, you don't need a drone with a long range or the ability to take photos from far away.

- Scaffolds, ladders, or other portable platforms: If the building is only one story high, or if you are only modeling a facade or other subset of a building, you may be able to get all the photos you need using a ladder or a platform with lockable wheels. You would, of course, need to make sure that the ground around 
the structure is flat, but this can get you good results with equipment that you may already have around.

Other costs associated with photogrammetry are software for stitching together your model and any expertise you need to help with the model or with sharing it in other formats. The Agisoft Metashape application is around $\$ 60$ for an educational license, and there are free or low cost methods too, though these may limit the number of photos you can use in your model or the number of models you can make. Some educational institutions may already pay for this for students, faculty, or staff. If you need the professional version of a photogrammetry application for complex (usually aerial) imagery or for making many models, expect to spend up to around $\$ 600$. If you are willing to learn to do this yourself, you may not have any external costs for expertise, but it is possible to hire people to help with the photogrammetric process, and may even save you money. For example, you can usually hire someone to take the aerial photographs for one building or building complex for less than the cost of buying a drone.

\section{Solid or Surface Modeling}

Dimensional projects that use solid or surface modeling have fairly low equipment costs, and instead require greater investment in labor or training. Many of the most popular modeling programs like Maya and Fusion 360 have free licenses for people associated with educational institutions that have all the necessary features. Most recent desktop computers can handle processing these models, though they may run slowly. If you will be doing a lot of modeling or you expect to have especially complex models, you may wish to seek out a computer with higher core processing speed (for interacting with your model) or more cores (for speeding up rendering), but you will still be fine with an ordinary consumer-market computer.

\section{If you are unable or unwilling to learn to use the modeling software yourself} and you need to hire someone to do it, be aware that these skills typically cost at least $\$ 40-\$ 50$ an hour for a freelancer, and will often require a lot of time as the kinds of accurate detail that matter in a humanities context are not as common in the storytelling applications of modeling that are more typical uses of these tools.

\section{Using \& Sharing 3D models}

Finally, there are the costs associated with using or sharing your model, no matter which process you use. If you just want to put your model online, you can usually do this for free through a platform like Sketchfab. If you already have a website, you can 
host your model there using free, open-source tools to help your audience use the model. In some cases, though, you may have files that are too large to upload to your website, so you may have to host the files somewhere else and pay a small amount.

If you are working on a virtual reality model, the primary costs to be aware of are the costs for your test equipment or equipment for your audience to use. VR headsets range from around \$10-\$15 at the cheap end (for something like Google Cardboard) to over $\$ 1000$ for top-level headsets with controllers.

The cost for 3d printing a model depends on how much material is needed to print it (based on size and how solid it is), the type of material, and a few other features that relate to the type of printer you need to use, like the tolerance for error. For most single-model projects, you would not want to buy your own printer, since you can print at many institutions for free or a low cost, and even the commercial cost of a model would be many degrees of magnitude smaller than the cost of buying a printer. Making your models hollow will keep the cost low, as will keeping the overall size down, and plastic-based materials are typically cheapest. If you do decide to buy a printer, they are available starting at around $\$ 200$ for a basic model, and plastic filament to use in them starts at around $\$ 30$ per kilogram. Printing a small single model in plastic through an online service can often be less than $\$ 10$.

Continue Reading: Timelines \& Workflows for Dimensional Projects 